\title{
ANTI CANCER STUDIES OF SELECTIVE MANNICH BASES BY IN SILICO METHOD
}

\author{
L. MURUGANANDAMa ${ }^{*}$, MAHESWARI R. ${ }^{\mathrm{a}}$ \\ aDepartment of Chemistry, Saranathan College of Engineering, Tiruchirappalli, India \\ Email: muruganandam-che@saranathan.ac.in
}

Received: 25 Dec 2017, Revised and Accepted: 05 Feb 2018

\begin{abstract}
Objective: To evaluate the anticancer activities of selective Mannich bases by in silico methods.
\end{abstract}

Methods: X-ray crystallographic structure of Estrogen receptor protein (PDB ID 2YAT) was downloaded from the protein data bank (PDB) and is docked with the target Mannich bases using Accelyrs Discovery Studio client version 2.5 software.

Results: Based on the in silico analysis results of the target compounds with standard drug tamoxifen, the best-docked compound is identified and its anticancer activity is confirmed by using in vitro MTS analysis using Raju and Jurkat cell lines.

Conclusion: The mannich base compound N-[(Diphenylamino) methyl] acetamide showed fourfold higher activity than standard drug tamoxifen, may be used to overcome the drug resistance of Estrogen receptor protein.

Keywords: Mannich bases, Docking, Estrogen, Anticancer

(C) 2018 The Authors. Published by Innovare Academic Sciences Pvt Ltd. This is an open access article under the CC BY license (http://creativecommons.org/licenses/by/4.0/) DOI: http://dx.doi.org/10.22159/ijcpr.2018v10i2.25877

\section{INTRODUCTION}

Docking, a computational tool often provides comprehensive insight into molecular mechanisms of biological processes [1]. The main objective of docking is to determine the best possible conformation of protein-ligand, protein-protein and/or another type of interactions with minimal energy [2]. Such studies give a path to explore novel products rapidly and economically that could be very specific for a particular target [3]. The relationship between chemical structure and biological activity is the basis for designing and synthesis of new drugs [4]. A huge number of papers related to Mannich bases are published in various pharmaceutical journals is the direct evidence of the application of these compounds [5]. They are beta amino ketones prepared by condensation of a compound with active hydrogen(s) with an amine (primary or secondary) and formaldehyde (any aldehyde) [6]. These bioactive leads are used for the synthesis of various clinically useful compounds containing aminoalkyl chain like cocaine, fluoxetine, atropine etc [7]. Mannich bases are known to possess potent activities like anti-microbial, antimalarial, anti-HIV, anti-cancer, anticonvulsant activities and so forth [8]. Mannich bases have remarkable biological potential which is remaining unexplored. However, our work would expectantly shed light on anticancer activities of specific Mannich bases.

Estrogens, natural hormones are important in sexual development and other body functions. Before menopause, they are produced mainly in the ovaries. After menopause, they are produced mainly in fat tissue. Women who begin menstruating early, or who start menopause late, produce more estrogen over their lifetimes and have a higher risk of breast cancer. A high level of estrogen is linked with increased risk of breast cancer which mediates its biological effects such as genesis, malignant progression, cell apoptosis and other important roles by binding to the Estrogen Receptor present in the breast cancer cells [9]. The Estrogen Receptor mainly exists in two forms: Estrogen Receptor alpha and Estrogen Receptor Beta. The major causes of breast cancer are identified by abnormal expression of Estrogen Receptor $\alpha$-positive affecting about $70 \%$ of the primary breast cancer patients [10]. The Estrogen Receptor alpha plays a pivotal role in controlling transcription of nuclear DNA necessary for mammary gland development [11] and it is also an essential factor for breast cancer signalling network [12]. It also regulates cell proliferation and differentiation through a paracrine mechanism [13] hence, the inhibition of Estrogen Receptor has become a major approach for preventing and treating breast cancer [14]. The drugs which are currently used, for the treatments of breast cancer interferes with either estrogen production or estrogen action which causes so many side effects such as blood clots, strokes, uterine cancer, or cataracts[15] The side effects of the currently used drug made us to explore an alternative approach to finding out new drug compound which are having anti-breast cancer So, we proposed to examine the anticancer activity of the synthesized Mannich bases against Estrogen receptor protein (PDB ID 2YAT) by molecular docking studies.

\section{MATERIALS AND METHODS}

\section{In silico anticancer screening}

The docking study was performed using Accelyrs Discovery Studio client version 2.5software. The X-ray crystallographic structure of Estrogen receptor protein (PDB ID 2YAT) was downloaded from the protein data bank (PDB). A grid-based molecular docking method, CDOCKER algorithm was used to dock the synthesized molecules into the protein active site. The designed structures were submitted to CHARMm (Chemistry at ARvard Macromolecular Mechanics) force field for structure refinement. All water molecules, bound inhibitor and other heteroatoms were removed from the macromolecule and polar hydrogen atoms were added. Energy minimization was carried out for all compounds using CHARMm force field to make stable conformation of the protein. A final minimization of the ligand in the rigid receptor using non-softened potential was performed. For each final pose, the CHARMm energy (interaction energy plus ligand strain) and the interaction energy alone were calculated. The poses were sorted by CHARMm energy and the top scoring (most negative, thus favorable to binding) poses.

\section{In vitro anticancer screening}

With the help of docking results obtained, the compound showing best anticancer activity was identified. In order to confirm the same, in vitro MTTS assay method was performed for the best-docked compound

\section{Cell lines}

Two cell lines manely Raji and Jukart were chosen. The number of cells is massively expanded in a minimal number of passages, and the cells are cryo-preserved to provide a consistent, long-term 
frozen stock for future use. Cells are grown and passaged in antibiotic-free growth medium to ensure the absence of microbial contaminants [16].

\section{Preparation and inoculation of cells}

Cells are separated into single cell suspensions by a gentle pipetting action, then counted using tryphan-blue exclusion on a Hemocytometer or by a counter [17]. After counting, dilutions are done to give the appropriate cell densities for inoculation onto the microtiter plates. Cells were inoculated in a volume of $100 \mu \mathrm{l}$ per well at densities between 5000 and 4000 cells per well. A $100 \mu \mathrm{l}$ aliquot of complete medium is added to cell-free wells. Prior to the inclusion of cell lines in the screening panel, their growth and compatibility were determined.

\section{Sample preparation}

All the samples were initially solubilized in phosphate buffer saline at $1: 1$ ratio and were filtered using membrane filter to avoid microbial contamination.

\section{MTT assay}

The assay was performed in a 96 well tissue culture plate (Greiner, U. S. A), using various negative controls and positive controls. After the addition of cell, media and solutions of complexes, the cultures were incubated in an incubator (TC2323, Shel lab, U. S. A) with 95\% air, 5\% $\mathrm{CO} 2$ and humidified atmosphere at $3 \mathrm{C}$ for $72 \mathrm{~h}$. The assay was monitored after $72 \mathrm{~h}$ based on spectrophotometer method by using an ELISA reader. After reading the plates, the $10 \mu \mathrm{l}$ tetrazolium compound MTT was added to the wells and incubated. After the incubation period, the MTT was reduced by mitochondrial dehydrogenase as a result of which the colour changed. Detergent SDS was added to the wells to solubilize the formazan crystals. The absorbance was read by making use of an ELISA reader at $570 \mathrm{~nm}$. The rate of tetrazolium reduction is directly proportional to the rate of cell proliferation [18].

\section{RESULTS AND DISCUSSION}

The docking results of Mannich bases and tamoxifen with Estrogen receptor protein (PDB ID 2YAT) were depicted in table 1 and their interactions with the active site of a target protein are given in fig. 1-7.

Table 1: CDOCKER energy results of docked compounds

\begin{tabular}{lll}
\hline S. No. & Compound name & CDOCKER energy \\
\hline 1 & $N$-[(Diphenylamino)methyl]acetamide & -29.2172 \\
2 & $N$-[(Diphenylamino)methyl]acrylamide & -27.1711 \\
3 & $N$-[Morpholino(phenyl)methyl]acetamide & -24.4197 \\
4 & [Morpholino(phenyl)methyl]benzamide & -23.1426 \\
5 & $N$-[Phenyl(pyrrolidin-1-yl)methyl]acetamide & -16.5583 \\
6 & $N$-[Phenyl(pyrrolidin-1-yl)methyl]benzamide & -12.7149 \\
7 & Tamoxifen & -7.62965 \\
\hline
\end{tabular}
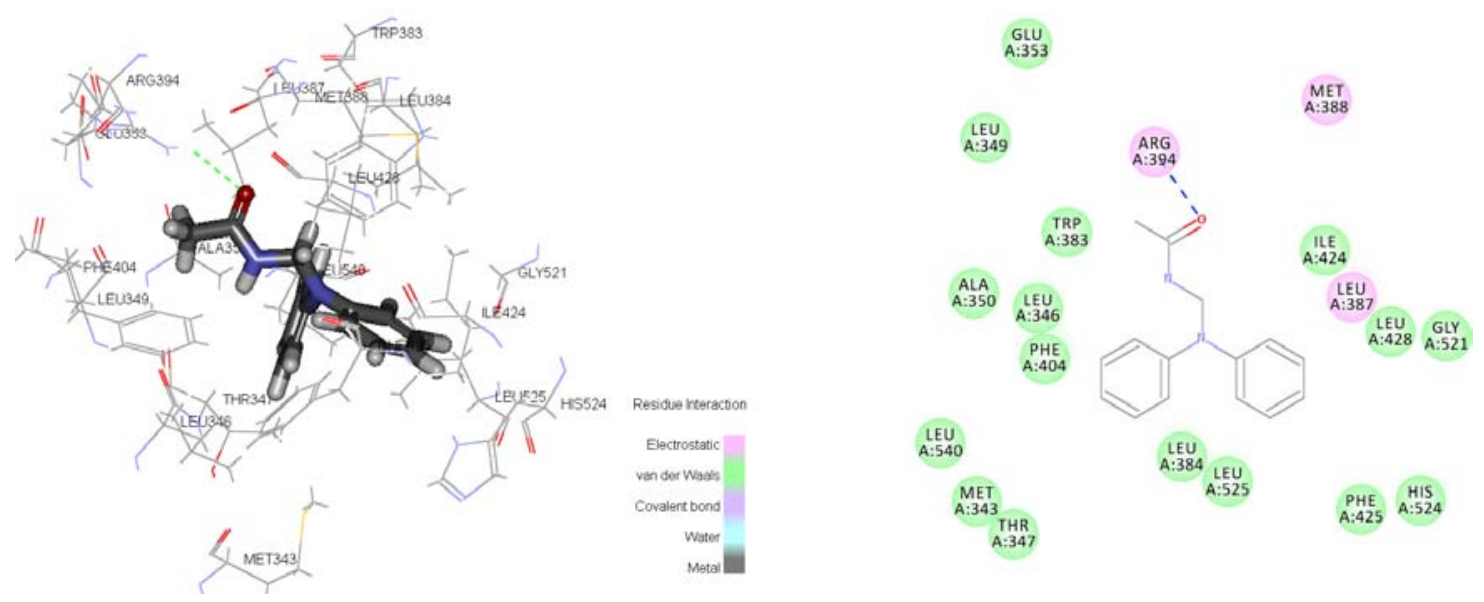

Fig. 1: Interaction details of $\mathrm{N}$-[(diphenylamino)methyl]acetamide with active site amino acids of 2YAT receptor
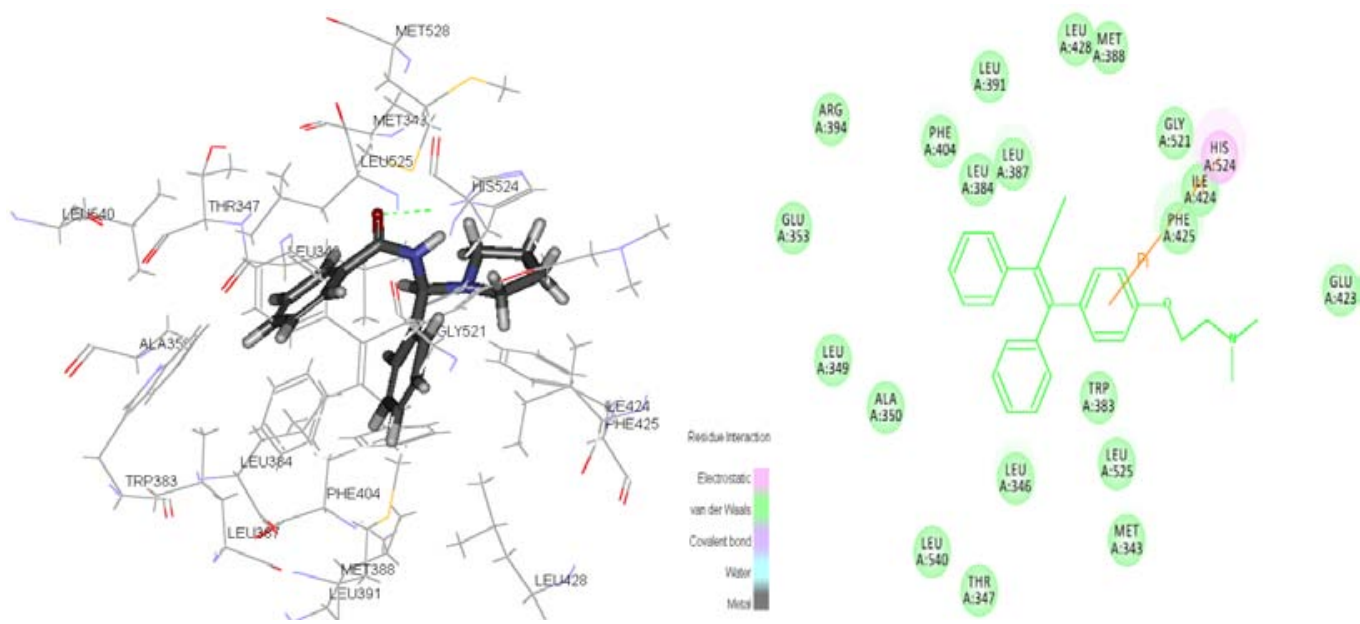

Fig. 2: Iinteraction details of $N$-[(diphenylamino)methyl]acrylamide with active site amino acids of 2YAT receptor 

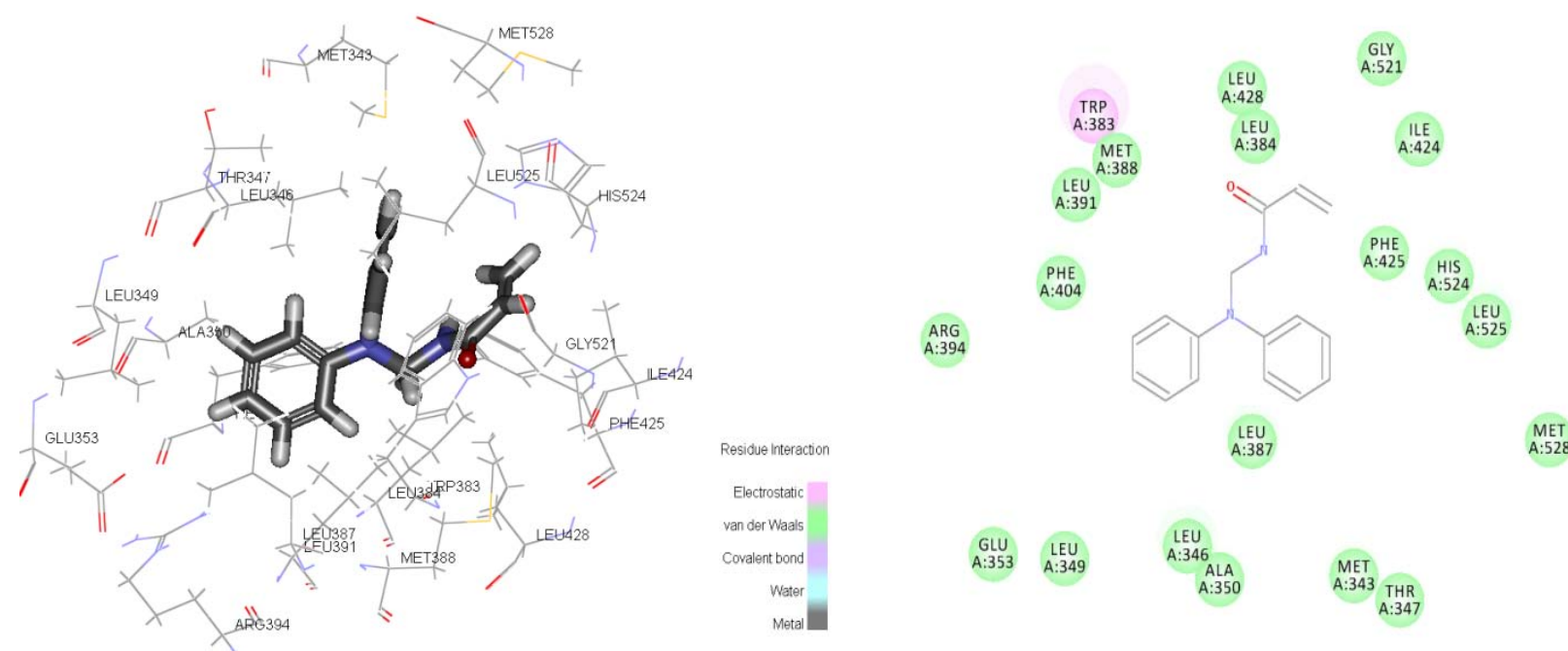

Fig. 3: Interaction details of $\mathrm{N}$-[morpholino(phenyl)methyl]acetamide with active site amino acids of 2YAT receptor
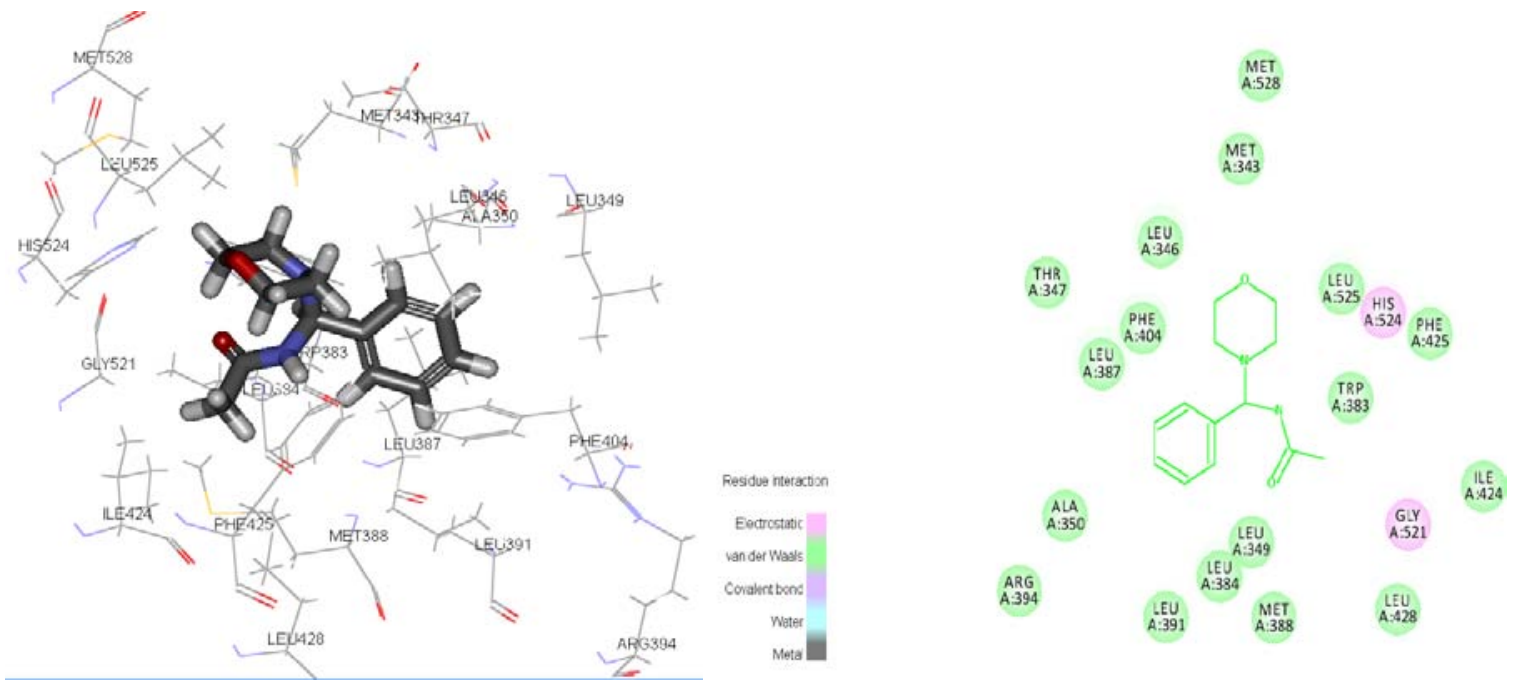

Fig. 4: Interaction details of [morpholino(phenyl)methyl]benzamide(MBB) with active site amino acids of 2YAT receptor
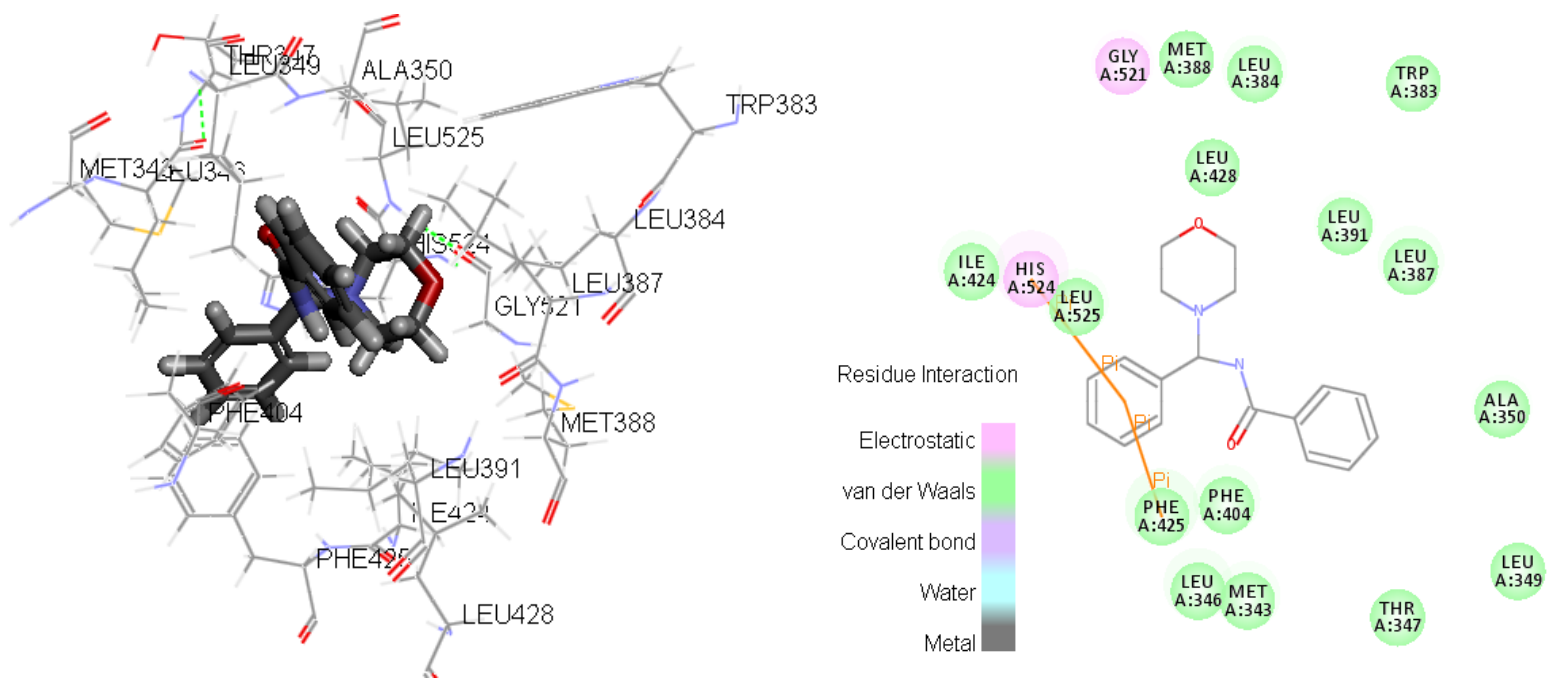

Fig. 5: Interaction details of N-[phenyl(pyrrolidin-1-yl)methyl]acetamide with active site amino acids of 2yat receptor 

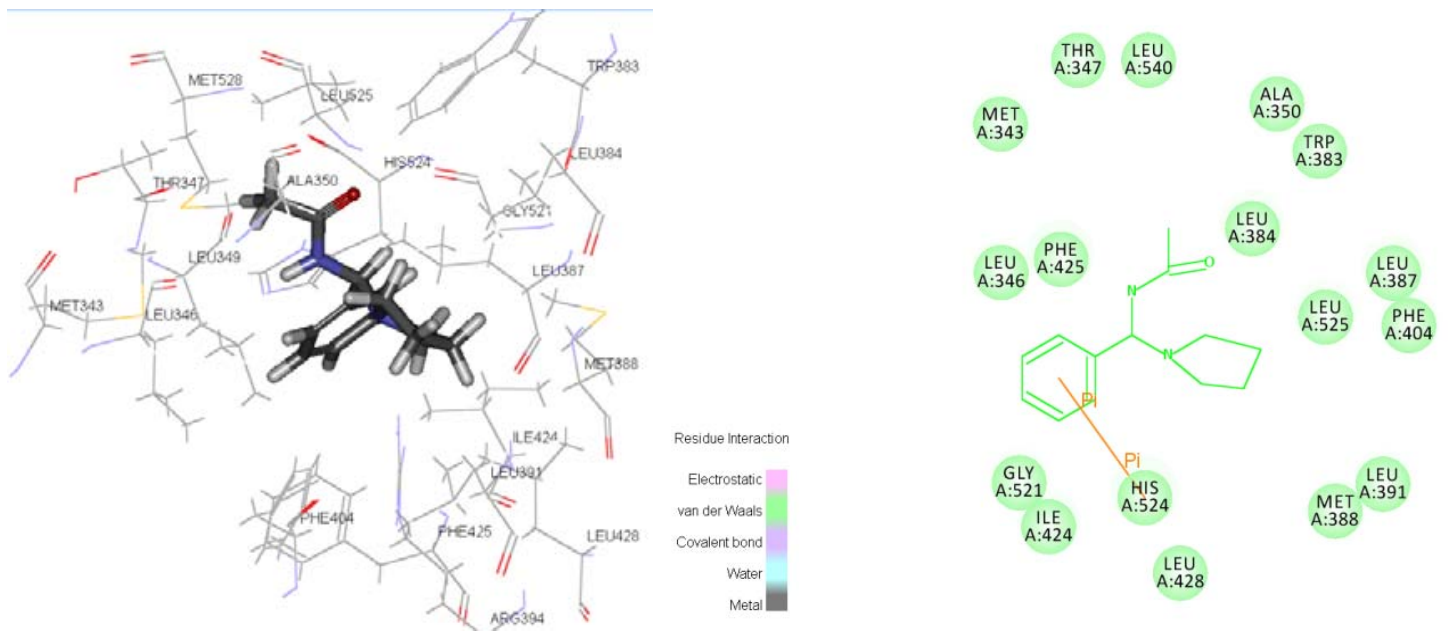

Fig. 6: Interaction details of $N$-[phenyl(pyrrolidin-1-yl)methyl]benzamide (PBB) with active site amino acids of 2YAT receptor
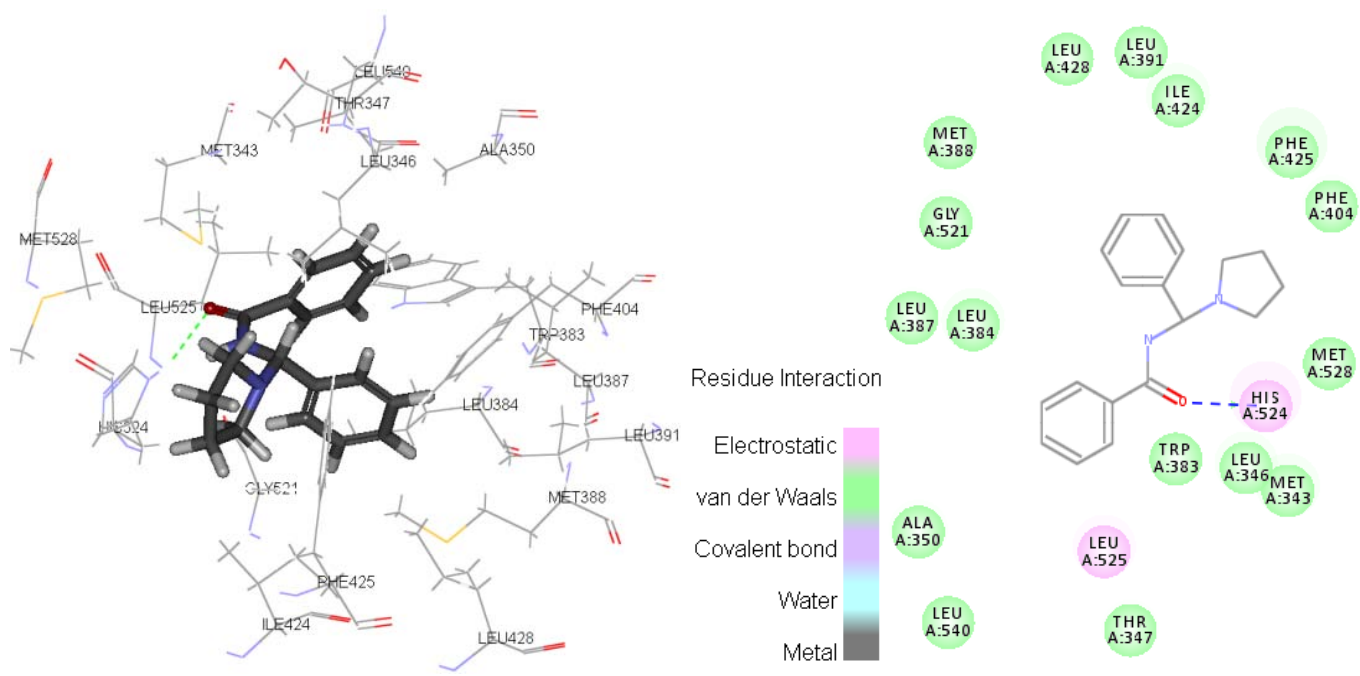

Fig. 7: Interaction details of tamoxifen with active site amino acids of 2YAT receptor

In this study, tamoxifen used as a standard ligand for comparison of docked compounds results. Tamoxifen is a nonsteroidal triphenylethylene derivative that binds to the estrogen receptor. It has both estrogenic and antiestrogenic actions, depending on the target tissue. It is strongly antiestrogenic on the mammary epithelium, hence its use in both the prevention and treatment of breast cancer; it is proestrogenic on the uterine epithelium, hence the current controversy regarding its safety in cancer prevention especially since an increased incidence of endometrial carcinoma has been found in women treated chronically with tamoxifen. Tamoxifen shown CDOCKER energy of-7.62965 $\mathrm{kcal} / \mathrm{mole}^{-1}$. It means the tamoxifen shown lower binding affinity with 2YAT protein compared with our compounds. There is no

\begin{abstract}
hydrogen bond involved between tamoxifen and receptor. Only one $\mathrm{Pi}-\mathrm{Pi}$ interaction was involved in between phenyl ring of the tamoxifen and His524. Additionally, several electrostatic and Van der waal's interaction are involved to make binding affinity of tamoxifen. The docking results of compounds with active site amino acids of 2YAT receptor were showed better binding interaction. In particular, the binding affinity of the $\mathrm{N}$-[(Diphenylamino) methyl] acetamide was found four-fold higher than standard. The ketone group of $\mathrm{N}-[($ Diphenylamino) methyl] acetamide molecule formed by one strong hydrogen bond (distance $1.98 \mathrm{~A}^{\circ}$ ) with $\mathrm{Arg} 394$ residue. To confirm the higher anticancer activity of $\mathrm{N}$-[(Diphenylamino) methyl] acetamide MTT assay was performed by in vitro methods and the results were given in table 2 .
\end{abstract}

Table 2: Anticancer activity against raji and jukart cell lines

\begin{tabular}{lll}
\hline Concentration & Raji cell line (a) & Jurkat cell line (b) $^{\text {(b) }}$ \\
\hline 25 & 0.19 & 0.23 \\
50 & 0.19 & 0.23 \\
100 & 0.19 & 0.22 \\
200 & 0.18 & 0.22 \\
400 & 0.18 & 0.21 \\
\hline
\end{tabular}

(a) Cell Control $(\mathrm{CC})=0.19 \mathrm{ng}$; (b) Cell Control $=0.23 \mathrm{ng}$, Higher absorbance values of the target compound at various concentrations indicate an increase in cells viability of the target compound. 


\section{CONCLUSION}

The mannich base compound $\mathrm{N}$-[(Diphenylamino) methyl] acetamide showed fourfold higher activity than standard drug tamoxifen, may be used to overcome the drug resistance of Estrogen receptor protein.

\section{AUTHORS CONTRIBUTIONS}

All the author have contributed equally

\section{CONFLICT OF INTERESTS}

\section{Declared none}

\section{REFERENCES}

1. Shreelekha Dutta, Prashant S Kharkar, Niteshkumar U Sahu, Aparna Khanna. Molecular docking prediction and in vitro studies elucidate anti-cancer activity of phytoestrogens. Life Sci 2017;185:73-84.

2. Guoshun Luo, Mingqi Chen, Weiting Lyu, Ruheng Zhao, Hua Xiang. Design, synthesis, biological evaluation and molecular docking studies of novel 3-aryl-4-anilino-2H-chromen-2-one derivatives targeting $\mathrm{ER} \alpha$ as anti-breasr cancer agents. Bioinorg Med Chem Lett 2017;27:2668-73.

3. Venumadhav Janganati, Jessica Ponder, Shraddha Thakkar, Craig T Jordan, Peter A Crooks. Succinamide derivatives of melampomagnolide B and their anti-cancer activities. Bioinorg Med Chem 2017;25:3694-705.

4. Lipinski CA, Lombardo F, Dominy BW, Feeney PJ. Experimental and computational approaches to estimate solubility and permeability in drug discovery and development settings. Adv Drug Delivery Rev 2001;46:3-26.

5. K Meenakshi, N Gopal, M Sarangapani. Synthesis, characterization and antimicrobial activity of some novel schiff and mannich bases of isatin. Int J Pharm Pharm Sci 2014;6:318-22.

6. Pandeya SN, Lakshmi VS, Pandey A. Biological activity of mannich bases. Indian J Pharm Sci 2003;65:213-22.

7. Gheorghe Roman. Mannich bases in medicinal chemistry and drug design. Eur J Med Chem 2015;89:743-816.
8. Suman Bala, Neha Sharma, Anu Kajal, Sunil Kamboj, Vipin Saini. Mannich bases: an important pharmacophore in the present scenario. Intl J Med Chem 2014. http://dx.doi.org/ 10.1155/ 2014/191072.

9. Jeyabaskar Suganya, Mahendran Radha, Devi Leimarembi Naorem, Marimuthu Nishandhini. In silico docking studies of selected flavonoids-natural healing agents against breast cancer. Asian Pac J Cancer Prev 2014;15:8155-9.

10. Alpha Kumaraswamy Naidu Chitrala, Suneetha Yeguvapalli. Prediction and analysis of ligands for estrogen-related receptor. Asian Pac J Cancer Prev 2013;14:2371-5.

11. Pete Donnell, Murad Banaji, Anca Marginean, Casian Pantea. CoNtRol: an open source framework for the analysis of chemical reaction networks. Bioinformatics 2014;30:1633-4.

12. Benson JR, Jatoi I. The global breast cancer burden. Future Oncol 2012;8:697-702.

13. Fuqua SA. The role of estrogen receptors in breast cancer metastasis. J Mammary Gland Biol Neoplasia 2001;6:407-17.

14. Izadi P, Noruzinia M, Fereidooni F, Nateghi MR. Association of poor prognosis subtypes of breast cancer with estrogen receptor alpha methylation in Iranian women. Asian Pac J Cancer Prev 2012;13:4113-7.

15. Lipinski CA, Lombardo F, Dominy BW, Feeney PJ. Experimental and computational approaches to estimate solubility and permeability in drug discovery and development settings. Adv Drug Delivery Rev 2001;46:3-26.

16. Idhayadhulla A, Surendra Kumar R, Jamal Abdul Nasser A, Selvin J, Aseer Manilal. Synthesis of some mannich base derivetives derivatives and their antimicrobial activity study. Arabian J Chem 2014;7:994-9.

17. Yan Li, Xiaoming Qiang, Li Luo, Xia Yang, Yong Deng Aurone. Mannich base derivatives as promising multifunctional agents with acetylcholinesterase inhibition, anti- $\beta$-amyloid aggregation and neuroprotective properties for the treatment of Alzheimer's disease. Eur J Med Chem 2017;126:762-75.

18. Mona Piplani, Harish Rajak, Prabodh Chander Sharma. Synthesis and characterization of N-Mannich based prodrugs of ciprofloxacin and norfloxacin: In vitro anthelmintic and cytotoxic evaluation. J Adv Res 2017;8:463-70. 